\title{
NoNIP: Natures of Negativity in Introductory Physics
}

\author{
Suzanne W. Brahmia, ${ }^{1}$ Alexis Olsho, ${ }^{1}$ Trevor I. Smith, ${ }^{2}$ and Andrew Boudreaux ${ }^{3}$ \\ ${ }^{1}$ Department of Physics, University of Washington, Box 351560, Seattle, WA, 98195-1560 \\ ${ }^{2}$ Department of Physics \& Astronomy, Rowan University, 201 Mullica Hill Rd., Glassboro, NJ 08028, USA \\ ${ }^{3}$ Department of Physics, Western Washington University, Bellingham, WA 98225
}

\begin{abstract}
Mathematical reasoning skills are a desired outcome of introductory physics courses, particularly calculusbased courses. Signed quantities are ubiquitous in physics, and sign carries important and varied meanings. Unlike physics experts, novices struggle with the many roles signed numbers can play in physics contexts; recent evidence shows that unresolved struggle carries over to subsequent physics courses. Mathematics education research literature documents cognitive challenges of conceptualizing negative numbers as mathematical objects-for experts, historically, and for novices as they learn. We add to the small but growing body of physics education research that focuses on student reasoning about signed quantities and the role of the negative sign in models. This paper contributes a framework for categorizing the various natures of the negative sign in physics contexts, modeled on the established natures of negativity in algebra from the mathematics education research community. We hope such a framework can facilitate innovation in methods and curricular activities to catalyze a deeper mathematical conceptualization of signed quantities in the introductory courses and beyond.
\end{abstract}

\section{INTRODUCTION}

The development of mathematical reasoning skills is an important goal in many introductory physics courses, particularly those geared toward students majoring in physics and engineering fields. The sign of a quantity carries significance; unlike physics experts, novices struggle to understand the many roles signed numbers can play in physics.

Negative pure numbers are more cognitively difficult mathematical objects than positive pure numbers for pre-college students [1]. Mathematics education researchers have isolated a variety of 'natures of negativity' fundamental to algebraic reasoning in the context of high school algebra that go beyond a 'position on a number line' nature [2-4]. These natures of negativity are a foundation for scientific quantification, as the mathematical properties of negative numbers represent natural processes and quantities. Physics education researchers report that most calculus-based introductory physics students struggle to make meaning of signed quantities outside of the number line context learned in elementary school, despite completing Calculus I and beyond in mathematics $[5,6]$. Developing flexibility with negative numbers is a known challenge in mathematics education. Reasoning about negative signs is a significant hurdle for physics students at the undergraduate level.

Few published studies have focused on negativity in the context of the mathematics used in physics courses. Studies conducted with upper division physics students reveal robust student difficulties [7-9]. Brahmia and Boudreaux constructed physics assessment items based on the natures of negativity from mathematics education research [10] and administered them to physics students in the introductory sequence of courses $[5,6,11]$. The authors report that students had difficulty reasoning about signed quantity in the contexts found in the curriculum (e.g. negative work, negative acceleration in one dimension), but had less trouble with the unary nature of negativity. (The unary nature of negativity is embodied by position on a number line.) The authors concluded that students have particular difficulty with signed scalar product quantities, commonly associating the sign of the scalar with the direction of one of the factor vectors, and suspect that science contexts overwhelm some students' conceptual facility with negativity. In addition, it is the fact that the quantity is signed that poses difficulty; students struggled to interpret the meaning of either a positive or negative signed quantity [6]. This work reveals that signed quantities present difficulties for students that are often not reconciled before completion of the introductory sequence, carrying over into upper division course work. The current study contributes to this body of work by introducing a framework for categorizing the natures of negativity in physics, analogous to the natures of negativity developed in the context of algebra. This should allow researchers to characterize and address the conceptualization of signed quantity in introductory physics.

\section{A MODEL OF THE NATURES OF NEGATIVITY}

The first generation of the natures of negativity for introductory physics was based on the natures of negativity described by mathematics education researcher Vlassis [10]. Two of the authors (Brahmia and Boudreaux) developed survey items-one survey question for each of the three natures in two contexts: mechanics quantities and E\&M. The first survey item probes student understanding of the unary nature of the negative sign, the second probes the symmetrical nature, and the third, the binary nature (see Table I). Table II presents all three mechanics items for reference.

Although we found that many uses of the negative sign typically found in introductory physics courses could be categorized using that existing map, using a mathematics-based sorting scheme frequently lost the nuances of the physics in the mathematics. For example, we found that both scalars and vectors might be placed in the same broad category, despite the physical importance in physics of distinguishing between vector and scalar quantities. Our intent was to encode both 
TABLE I. A map of the different uses of the negative sign in elementary algebra; the triple nature of the negative sign [10]

\begin{tabular}{ccc}
\hline \hline Unary (Structural signifier) & Symmetrical (Operational signifier) & Binary (Operational signifier) \\
\hline Subtrahend & Taking opposite of, or inverting, the operation & Completing \\
Relative number & & Taking away \\
Isolated number & & Difference between two numbers \\
Formal concept of negative number & Movements on the number line \\
\hline \hline
\end{tabular}

TABLE II. Examples of questions representing different uses of the negative sign in the context of introductory mechanics

\begin{tabular}{|c|c|c|}
\hline Unary & Symmetrical & Binary \\
\hline structural signifier & operational signifier & operational signifier \\
\hline Direction of a vector component & $\begin{array}{l}\text { Signifies work results in decreasing } \\
\text { the system energy, not increasing it }\end{array}$ & Position relative to an origin \\
\hline $\begin{array}{l}\text { An object moves along the } \mathrm{x} \text {-axis, and } \\
\text { the acceleration is measured to be } \\
a_{x}=-8 \mathrm{~m} / \mathrm{s}^{2} \text {. Describe in your } \\
\text { own words the meaning of the } \\
\text { negative sign in the mathematical } \\
\text { statement " } a_{x}=-8 \mathrm{~m} / \mathrm{s}^{2} . \text { " }\end{array}$ & $\begin{array}{l}\text { A hand exerts a horizontal force on a block as the } \\
\text { block moves on a frictionless horizontal surface. } \\
\text { For a particular interval of the motion, the work } W \\
\text { done by the hand is } W=-2.7 \mathrm{~J} \text {. Describe in } \\
\text { your own words the meaning of the negative } \\
\text { sign in the mathematical statement " } W=-2.7 \mathrm{~J} \text {." }\end{array}$ & $\begin{array}{l}\text { A cart is moving along the } \mathrm{x} \text {-axis. At a } \\
\text { specific instant, the cart is at position } \\
x=-7 \mathrm{~m} \text {. Describe in your own words } \\
\text { the meaning of the negative sign in the } \\
\text { mathematical statement " } x=-7 \mathrm{~m} . \text { " }\end{array}$ \\
\hline
\end{tabular}

physical and mathematical meaning with our map; we started from scratch keeping the physics as our primary guide.

We (Brahmia and Olsho) employed a modified card-sorting task for our second attempt for physics quantities and relationships typically introduced in introductory physics. Categories were created based on overarching similarities. We created several sub-categories for each main category, largely to account for nuances in physical meaning. We determined three basic categories: Direction (D), Opposition $(O)$, and Change (Ch). A fourth category, Compound $(\mathrm{Co})$ was added for instances when multiple meanings are assigned to the negative sign in a single expression or concept. Table III shows the results of this effort to create a map of the natures of negativity in introductory physics (NoNIP). We surveyed introductory physics textbooks to help ensure that the NoNIP is a valid categorization scheme for typical uses of the negative sign in introductory physics. This involved looking at all instances of use of negative signs. With one notable exception (negative exponentials), we found that all uses of the negative sign could be categorized satisfactorily using the NoNIP. The omission of negative exponentials is intentional, as we do not yet have adequate research on student understanding of negative exponentials and logarithms in physics contexts. We also validated the NoNIP with interviews with mathematics and physics experts. Interview subjects were asked to comment on the appropriateness of the map in the context of introductory physics, as well as any uses of the negative sign that were not compatible with the NoNIP framework. Expert comments were overwhelmingly supportive and resulted in a number of small improvements in the NoNIP. We plan on doing focus groups with physics instructors to determine the extent to which the NoNIP can inform their understanding of issues with negativity in student discourse and problem-solving.

The Direction category is used largely for vector quanti- ties. We differentiate between 1. Location (for which the sign tells us the position relative to an origin), 2. Direction of motion (typically used for a vector component, where sign indicates direction of motion relative to a coordinate system), and 3. Other vector quantities (where the sign of a vector component tells us the direction of that component relative to a coordinate system, but when motion is not an intrinsic quality of the vector quantity). Finally, we consider 4. Above/below reference for scalar quantities such as electric potential difference and temperature where the zero of the quantity is an arbitrary reference point.

For the category Opposition, we consider quantities for which a negative sign implies opposite direction or relationship. The use of sign to denote a type of object (charge) falls under this category in the subcategory 1. Opposite type. Positive and negative charge are 'opposite' types of charge, and obey the mathematical relationship of $+q+(-q)=0$ (adding equal amounts of opposite types of charge gives system with 0 net charge). For the the subcategory 2. Opposes, we consider scalar and vector relationships between quantities that indicate that the quantities oppose each other in direction or change, such as members of a Newton's Third Law force-pair or the relationship between flux and emf described by Faraday's Law. We also include scalar product quantities such as $\Phi_{E}, \Phi_{B}$, and $W$, in which a negative quantity indicates opposition (of direction) between the factor vectors.

The category Change encompasses both the meaning of the sign of the change of a quantity (such as energy or entropy) as well as the negative sign as an operator that signifies a change in a quantity. As an operator, the negative sign may signify a physical removal or decrease of a quantity, as in the removal of charge from a system. We may also use the negative sign to signify that we are taking a difference between two quantities (as in determining the change of a quantity.) 
TABLE III. Preliminary map of the natures of negativity in introductory-level physics

\begin{tabular}{|c|c|c|c|}
\hline (D) Direction & (O) Opposition & (Ch) Change & (Co) Compound \\
\hline 1. Location & 1. Opposite type & 1. System scalar quant. & 1. Scalar, vector $\Delta$ \\
\hline$x$ & $Q($ charge $)$ & $\Delta K, \Delta E$ & $\Delta E=E_{f}-E_{i}$ \\
\hline 2. Dir. of motion & 2. Opposes & $\Delta S$ & $\Delta \vec{p}=\vec{p}_{f}-\vec{p}_{i}$ \\
\hline$v_{x}, \Delta \vec{x}$ & $\vec{F}_{12}=-\vec{F}_{21}$ & 2. Removal (operator) & $\Delta Q$ \\
\hline$p_{x}$ & $\vec{F}=-\vec{\nabla} U$ & $0-(-5 \mu C)$ & 2. Scalar rates of change \\
\hline 3. Other vec. quant. comp & $\mathscr{E}=-\frac{d \Phi_{B}}{d t}$ & 3. Difference (operator) & $\frac{d \Phi}{d t}$ \\
\hline$E_{x}, B_{x}$ & $F=-k x$ & $E_{f}-E_{i}$ & 3. Base + change \\
\hline$F_{x}, \vec{\tau}$ & 3. Scalar products & $\overrightarrow{p_{f}}-\vec{p}_{i}$ & $\phi+\frac{d \phi}{d t} t$ \\
\hline$a_{x}$ & $W=\overrightarrow{\vec{F}} \cdot \Delta \vec{x}$ & & $\vec{v}+\vec{a} t$ \\
\hline$\Delta p_{x}, \Delta v_{x}$ & $\Phi=\vec{B} \cdot \vec{A}$ & & 4. Products $f(x) d x$ \\
\hline 4. Above/below reference & & & $E(r) d r$ \\
\hline$T$ (temperature) & & & $P(V) d V$ \\
\hline$V($ voltage $)$ & & & $\begin{array}{l}\text { 5. Models } \\
W_{n e t, e x t}=\Delta E \\
\vec{F}_{n e t}=m \vec{a}\end{array}$ \\
\hline
\end{tabular}

Finally, the Compound category covers instances when the negative sign spans more than one category, or that require one to 'keep track' of several signs when making sense of a quantity or relationship. For example, as in subcategory 1. Scalar, Vector $\Delta$, when students are asked to calculate a change in a quantity such as energy or momentum, they must first account for the signs of the initial and final quantities, and then successfully subtract one from the other; then they may be expected to make sense of the result. Similarly, students may be expected to account for multiple negative signs when considering 2. Scalar rates of change, 3. Base + change (base quantities that are increased or decreased by the addition of a change), and 4. Products $f(x) d x$ (products of integrands and differentials). We also include in this category 5. Models, to account for models that require sensemaking of a negative sign. The Work-Energy Theorem, where the sign of $W_{\text {net,ext }}$ indicates whether a system gains or loses mechanical energy, is an example of such a model.

\section{APPLYING THE FRAMEWORK}

In this section, we view recently published studies in three diverse areas of physics using the NoNIP as an analytical lens.

Bajracharya, Wemyss and Thompson (2012) investigated upper-division student understanding of integration in the context of definite integrals commonly found in introductory physics [7]. Their results suggest difficulties with the criteria that determine the sign of a definite integral. Students struggle with the concepts of a negative area-under-the-curve, and negative directions of single-variable integration. The difficulties the authors describe can be seen through the lens of NoNIP as difficulty with the product of the integrand, $f(x)$, and the differential, $d x$ (Co.4 in NoNIP). The negativity of the integrand (D in NoNIP) was less of a challenge for the students in this study than was the notion of a negative differ- ential (Ch in NoNIP), seen throughout physics.

A study conducted by Hayes and Wittmann (2010) situated in the context of junior level mechanics, investigates the negative signs and quantities associated with the equation of motion of an object thrown downward, with non-negligible air resistance [8]. The interviewed student struggles with treating one dimensional acceleration as a signed quantity, and feels there should be an additional negative sign included to indicate that the acceleration is 'negative,' or opposing the motion. The authors explain student difficulties with negativity using implicit and explicit 'minus' signs. They conclude that the multiple natures of the negative sign are a source of cognitive conflict that manifests as sensemaking about 'outer and inner minus.' An 'outer minus' is a negative sign that is assigned, for example, by choice of coordinate system; an 'inner minus' is one that is associated with variables that may be negative. Through the lens of NoNIP, "minus" is an operator, and negative signs are used to represent many mathematical objects and relationships in physics. In the framework of NoNIP, the student struggles with D.3 and D. 2 in the contexts of one-dimensional acceleration and velocity. The negative sign that modifies the $c v$ term is used as 0.2 , to indicate that the force is in the opposite direction to the velocity. Combining these terms, the students struggle to make sense of the equation of motion. The cognitive load of negativity associated with the individual terms contribute to a higher-level struggle of making physical sense (Co.5).

In their study of negativity in junior level E\&M, Huynh and Sayre (2018) describe the in-the-moment thinking of a student solving for the direction of the near electric field of an electric dipole along the dipole axis [9]. The authors detail the student's development of an increasingly blended approach, situated in a mental space informed by both mathematical and physical concepts. The student starts reasoning about the direction of the field by unknowingly combining multiple natures of negativity into one, using the cancelling procedure 
that 'two negatives make a positive' without considering the source of each negative sign. In Coulomb's law, signs are associated with the charges, the unit vector, and the field direction. Combining the signs is a common approach that focuses on the multiplicative rules of signed numbers rather than the physical meaning of the signs. Next the student rarifies his approach as he considers more carefully the natures of negativity in the context of the problem. Using NoNIP, we can see evidence of the student first conflating the natures superficially, "...he decides to absorb the destructive meaning...into the opposite meaning...and changes the second negative sign to a plus sign...however he didn't consider the...relative direction...leading to...the opposite sign of the correct answer." Then he slows his thinking, recognizing the unit and electric field vectors (D.2 and D.3) as sources of negative signs. After reconciling this, he struggles with O.2. The authors claim that the student "has successfully affiliated the sign's meaning to the relative direction...electric fields and x-hat." The authors conclude, and we agree, that the most sophisticated challenge occurs when these natures are combined-three natures of the negative sign must be understood in the context of a single equation (Co.5). "[T]o successfully deal with the algebraic signs, the student has to recognize these different [blends] and select among them," where the authors use "blend" to describe the physical and mathematical meanings of the signs combined. This example illustrates the challenges associated with reasoning about the natures of negativity even for strong majors, and lends plausibility to the NoNIP model being representative of student thinking.

\section{IMPLICATIONS FOR INSTRUCTION}

We note that all three of these studies are conducted with physics majors in upper division courses. Difficulties are embedded in natures of negativity that can and should be explicitly addressed in the context of introductory physics.

As we dig deeper into this work, we see many contexts and opportunities to make explicit our thinking with our students regarding signed quantities, as we hope other instructors will too. We suggest that instructors familiarize themselves with the many jobs that the negative sign does in our courses, and help students recognize the same. The NoNIP framework can help. We note that sign and operation are often conflated using an equals sign (e.g., $5+(-3)=5-3$ ); unsigned numbers are assumed positive. Adding a negative quantity and subtracting a positive one often have different meanings in physics contexts (e.g., removing electrons). Although these operations yield the same arithmetic results, conflating them may lead students to struggle with the distinctions between sign and operation. We suggest using the term "minus" for the operation of subtraction, and the term "negative sign" to describe the symbol. We note that some of the work cited in this paper does not follow this recommendation, notably Vlassis (2004) and Hayes \& Wittman (2010) [8, 10]. Aligning the positive coordinate axis with the direction of motion eliminates the need for signed quantities when discussing velocity. This choice, however, could be a missed opportunity to distinguish between orientation (along a particular axis) and sense (positive or negative). The opposite coordinate choice can prime students to consider the signed nature of position, velocity, and subsequent vector quantities they encounter.

In addition to enriching physics learning, a focus on natures of negativity in physics contexts can also enrich the corequisite mathematics learning. Researchers report on contexts in which physics helps mathematics students make sense of negativity in calculus [7, 12]. We suggest that there is a symbiotic cognition possible in which both mathematics and physics learning can be enriched by conceptualization of the other. We present NoNIP as a representation of signed quantity providing a step in that direction.

\section{ACKNOWLEDGMENTS}

We thank Peter Shaffer for help with data collection informing this work, and the Physics Education Group at the University of Washington for discussion and feedback. We thank Roy Montalvo at Rutgers University for his software innovation for smooth data collection.
[1] J. P. Bishop, L. L. Lamb, R. A. Philipp, I. Whitacre, B. P. Schappelle, and M. L. Lewis, Journal for Research in Mathematics Education 45, 19 (2014).

[2] A. Gallardo and T. Rojano, Proceedings of the XVI International Group for the Psychology of Mathematics Education, North American Chapter 1, 265 (1994).

[3] P. W. Thompson and T. Dreyfus, Journal for Research in Mathematics Education, 115 (1988).

[4] T. Nunes, Schools, mathematics, and the world of reality , 61 (1993).

[5] S. Brahmia and A. Boudreaux, in Proceedings of the 19th Annual Conference of RUME (2016) p. 79.

[6] S. Brahmia and A. Boudreaux, in Proceedings of the 20th An- nual Conference on RUME (San Diego, CA, 2017).

[7] R. R. Bajracharya, T. M. Wemyss, and J. R. Thompson, AIP Conference Proceedings 1413, 111 (2012).

[8] K. Hayes and M. C. Wittmann, The Physics Teacher 48, 246 (2010).

[9] T. Huynh and E. C. Sayre, arXiv preprint arXiv:1803.01447 (2018).

[10] J. Vlassis, Learning and instruction 14, 469 (2004).

[11] S. W. Brahmia, in Physics Education Research Conference 2017, PERC (2017) pp. 64-67.

[12] V. Sealy and J. Thompson, in Proc. of the 19th Annu. Conf. on RUME (2016). 